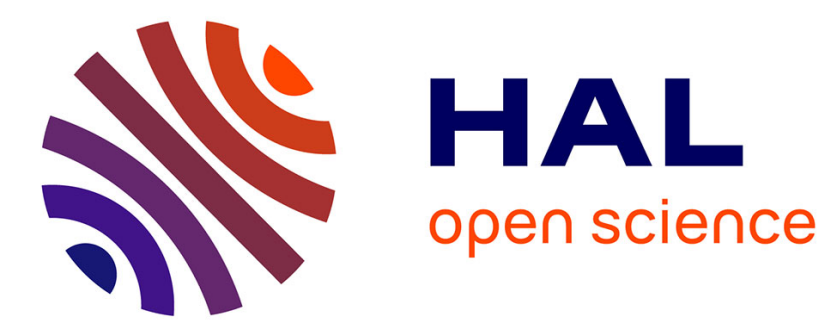

\title{
Experimental evidence of high spatial confinement of elastic energy in a phononic cantilever
}

Etienne Coffy, Sébastien Euphrasie, Pascal Vairac, Abdelkrim Khelif

\section{To cite this version:}

Etienne Coffy, Sébastien Euphrasie, Pascal Vairac, Abdelkrim Khelif. Experimental evidence of high spatial confinement of elastic energy in a phononic cantilever. Applied Physics Letters, 2021, 119 (20), pp.203501 (6). hal-03549394

\section{HAL Id: hal-03549394 \\ https://hal.science/hal-03549394}

Submitted on 31 Jan 2022

HAL is a multi-disciplinary open access archive for the deposit and dissemination of scientific research documents, whether they are published or not. The documents may come from teaching and research institutions in France or abroad, or from public or private research centers.
L'archive ouverte pluridisciplinaire HAL, est destinée au dépôt et à la diffusion de documents scientifiques de niveau recherche, publiés ou non, émanant des établissements d'enseignement et de recherche français ou étrangers, des laboratoires publics ou privés. 


\title{
Experimental evidence of high spatial confinement of elastic energy in a phononic cantilever
}

Etienne Coffy, Sébastien Euphrasie*, Pascal Vairac and Abdelkrim Khelif

FEMTO-ST Institute, Université de Bourgogne Franche-Comté, UMR CNRS 6174, 15B Av. des Montboucons, F-25030 Besançon, France

\begin{abstract}
We report on experimental high spatial confinement of elastic energy in a silicon phononic cantilever for which the quality factor of a higher-order flexural resonance is increased by a factor of 27 (from $\mathrm{Q} \sim 80$ to $\mathrm{Q} \sim 2130$ ) with the use of a 3-row phononic crystal (PnC) strip. As shown by numerical simulations performed with the finite element method, the PnC both reduces anchor loss and confines elastic energy inside the cantilever. The $\mathrm{PnC}$ and the cantilever are fabricated with standard clean room techniques on a SOI (silicon on insulator) substrate. Optical measurements of the out-of-plane displacements are performed with a laser scanning interferometer in a frequency range around $2 \mathrm{MHz}$.
\end{abstract}

Phononic crystals (PnC) are artificial periodic structures that possess band gaps, that is to say frequency ranges where no acoustic or elastic waves can propagate, whatever the polarization $[1,2]$. PnC cover a wide range of frequencies ranging from low frequencies for seismic engineering [3] to microwaves or higher frequencies with MicroElectroMechanical Systems (MEMS) or NanoElectroMechanical Systems (NEMS) [4]. Applications of PnC include wave filtering [5, 6], sound or vibration mitigation [7], sound collimation [8] or insulation [9, 10], elastic or acoustic waveguiding [11, 12], focusing [13], among others. PnC have also been used to reduce anchor loss to confine the energy into a resonator and increase the quality factor $\mathrm{Q}$ of the resonant mode [14-22].

Quality factor Q is defined as the ratio of stored energy to energy dissipation. Various loss mechanisms can exist simultaneously in MEMS. Anchors loss (anch) accounts for energy leakage from the anchors. Air damping (air) accounts for air friction and for all dissipative phenomena due to the ambient air. Thermoelastic damping (TED) originates from the irreversible heat flux caused by temperature gradients due to the alternating spatial variations of strain and can dominate in flexural-mode resonators [17]. Akhiezer damping (Akh) takes place because of the heat flow between different phonon modes and sets a fundamental limit for Q. Other types of losses, such as interface losses, also exist. Q can then be estimated from the following equation:

$$
\frac{1}{Q}=\frac{1}{Q_{\text {anch }}}+\frac{1}{Q_{\text {air }}}+\frac{1}{Q_{T E D}}+\frac{1}{Q_{A k h}}+\frac{1}{Q_{\text {others }}} .
$$

When anchor losses dominate, Q can be increased by using PnC with a band gap containing the resonant frequency so that energy leakage is prevented. Sorenson et al. [15] used a PnC strip (one dimensional $\mathrm{PnC}$ ) of coupled rings, six periods long, to obtain an experimental mean increase of Q by a factor of 2. Feng et al. [16] obtained an experimental increase of Q by $34 \%$ considering a 2-period PnC strip; the unit cell of the strip had large quarter cylinders removed at each corner. Lee et al. [17] used a 4-period PnC tether of rings and experimentally achieved an increase of $\mathrm{Q}$ by a factor of 3. They also proposed a 2 dimensional PnC of rings as a reflector positioned after normal tethers and obtained an experimental increase by a factor of 2 with an air hole PnC [18] and a factor of 4.2 with solid disk-shaped PnC [22]. The authors of ref. [19] used a planar ring-shaped $\mathrm{PnC}$ as anchor boundaries to increase the robustness and obtained an experimental enhancement of Q by a factor of 3. Gokhale et al. [20] nearly removed all the anchor loss with a PnC strip made of 5 periods of square blocks connected by narrow tethers and operating at $282 \mathrm{MHz}$. They obtained an increase of Q by more than 2 compared to $\lambda / 8$ narrow tethers. Recently, Bao et al. [21] proposed a five stages PnC structure to obtain 
experimentally a very high improvement of Q by a factor of 19.4. All these results can find potential applications in MEMS devices as well as in scanning microscopes, such as atomic force microscopes (AFM), which can exhibit a higher sensitivity if the vibration energy is confined to the tip and the quality factor of the resonance is increased.

In this study, we design a PnC strip to reduce anchor loss of a cantilever vibrating in its sixth order flexural mode in air (the length of the cantilever is roughly five half-wavelengths and a quarter of the wavelength). This mode has been chosen because it is the first flexural mode in the band gap. The other modes were not deemed as interesting for practical applications. Choosing other dimensions for the cantilever would lead to other interesting modes in the band gap. In particular, a cantilever far smaller would have the first flexural mode in the band gap but the measurements would have been more difficult. The PnC strip and the cantilever are made from a SOI (Silicon on Insulator) wafer with MEMS techniques. Simulations with the finite element method (FEM) show a large complete band gap and the effective reduction of anchor loss. Optical measurement of the out-of-plane displacements prove an increase of the quality factor Q by a factor of 27 at $2 \mathrm{MHz}$, from 80 to 2130 .

The designed cantilever is made of a (100)-oriented silicon ( $\mathrm{Si}$ ) beam with dimensions 2000 $\mu \mathrm{m} \times 600 \mu \mathrm{m} \times 100 \mu \mathrm{m}$. Its sixth order flexural mode appears around $2 \mathrm{MHz}$ (depending on the anchor). The PnC strip structure consists of a periodic array of silicon pillars fixed to a tailored silicon strip (see figures $1 \mathrm{a} \& 1 \mathrm{~b}$ ). Previously, we reported that similar structures [2324] fabricated with tungsten (W) pillars instead of silicon ones possess very large phononic band gaps (with an experimental gap-to-midgap ratio of 94\%). Losing the mechanical contrast between $\mathrm{Si}$ and $\mathrm{W}$ reduces the gap-to-midgap ratio. However, it simplifies fabrication and enables mass production with standard CMOS compatible MEMS techniques. The device we characterize experimentally is made from a SOI wafer composed of a $625 \mu \mathrm{m}$ thick (100)oriented Si substrate, a $1 \mu \mathrm{m}$ thick $\mathrm{SiO}_{2}$ layer and a $100 \mu \mathrm{m}$ thick (100)-oriented Si layer. Photolithography and deep reactive ion etching (DRIE) are used to pattern the cantilever and the PnC strip from the top of the SOI wafer. Subsequently, the pillars are drilled from the back side of the SOI wafer. Buffered hydrofluoric acid (BHF) finally removes the $\mathrm{SiO}_{2}$ everywhere except under the pillars.

Figure 1.c depicts the unit cell of the PnC strip. The lattice constant $a$ is $1000 \mu \mathrm{m}$; the thickness of the strip $b$ and of the $\mathrm{SiO}_{2}$ thickness $h_{\mathrm{SiO} 2}$ are respectively 100 and $1 \mu \mathrm{m}$. The notches in the strip have a diameter $d_{H}=400 \mu \mathrm{m}$. The pillars are $h_{P}=625 \mu \mathrm{m}$ tall and have a diameter $d_{P}=$ $900 \mu \mathrm{m}$. Simulations in this paper are performed using the finite element method (FEM) with the commercial software COMSOL Multiphysics. The phononic band structure (cf. fig. 1.d) is calculated by applying the Bloch-Floquet theorem and assuming an infinite periodic strip. The color scale indicates the out-of-plane $(\mathrm{z})$ polarization content normalized to the total displacement. The main band gap ranges from 1740 to $3180 \mathrm{kHz}$, which corresponds to a gapto-midgap ratio of $59 \%$. Removing or varying the thickness of the $\mathrm{SiO}_{2}$ layer does not significantly change the band structure. The sixth order flexural mode of the cantilever is situated inside the band gap and therefore its anchor losses are expected to decrease.

The vibrations of two samples were studied. The first sample $\left(D_{s}\right)$, which can be considered as a reference, consists of the cantilever directly anchored to the substrate. The second sample $\left(\mathrm{D}_{\mathrm{PnC}}\right)$ consists of the cantilever linked to the substrate through a PnC strip of 3 periods and a square with dimensions $\mathrm{a} \times \mathrm{a} \times \mathrm{t}(1000 \mu \mathrm{m} \times 1000 \mu \mathrm{m} \times 100 \mu \mathrm{m})$, as shown in figure $1 \mathrm{a}$. The samples were set into motion with a piezoelectric ceramic, lead zirconate titanate (PZT), located above the base of the devices (see fig. 1.b). Figure 2 presents the whole spectrum of the mean out-of-plane absolute displacement for the two samples, measured from $100 \mathrm{kHz}$ to $5 \mathrm{MHz}$. It was obtained with a laser Doppler vibrometer system (Polytec MSA 500) under white noise excitation. The spatial scan of the side opposite to the pillars (assuming the laser coming from 
direction $+\mathrm{z}$ ) is performed with a spatial step of $50 \mu \mathrm{m}$. The experimental band gap is indicated by the green area in fig. 2. It is slightly down-shifted compared to the theoretical band gap of fig. 1d. Tolerances over the SOI nominal thicknesses (in particular $\pm 5 \mu \mathrm{m}$ for the $100 \mu \mathrm{m}$ layer which is the most sensitive) and fabrication imperfections (a few micrometers in particular in the notches diameter) do not completely explain this shift. Nonetheless, the band gap start frequency is difficult to precisely define because of the lack of strongly exited modes at this frequency. The resonances in $\mathrm{DPnC}_{\mathrm{PnC}}$ are few and are well defined, whereas there are more resonances for $\mathrm{D}_{\mathrm{s}}$. The latter resonances are not well defined because of their interference. We considered the first flexural mode that is located inside the band gap. It corresponds to the sixth one and is indicated by the blue and the red arrows in figure 2 . We attribute the frequency shift of that mode observed for samples $D_{s}$ and $D_{P n C}$ to the difference in anchor. Optical measurements of the out-of-plane displacement around the resonant frequencies are performed using a homemade laser scanning interferometer [25-26]. An alternative voltage of $1.5 \mathrm{~V}$ peak to peak is applied through the electrodes of the PZT transducer with a frequency sweep. The spatial scan is performed with a $500 \mu \mathrm{m}$ step. Figure 3a presents the mean out-of-plane absolute displacement averaged over the whole cantilever for the two samples. The vibration amplitude for sample $\mathrm{D}_{\mathrm{s}}$ (without the PnC strip) exhibits a resonance frequency of $2.215 \mathrm{MHz}$ with a quality factor of $80 \pm 10$. The response for sample $\mathrm{D}_{\mathrm{PnC}}$ (with the PnC strip) exhibits a resonance frequency of $2.021 \mathrm{MHz}$ with a quality factor of $2130 \pm 10$. The response for $D_{P n C}$ fits well with the square root of a Lorentzian function:

$$
z(f)=\frac{z_{\max }}{\sqrt{1+\left(2 Q\left(f / f_{0}-1\right)\right)^{2}}},
$$

where $\mathrm{f}$ is the frequency, $\mathrm{f}_{0}$ the central frequency, $\mathrm{z}$ the out-of-plane displacement and $\mathrm{z}_{\max }$ its maximum. This is not the case for the response for $D_{s}$, because of interferences with adjacent modes. Figure $3 b$ (respectively fig. 3c) presents the map of the out-of-plane displacement for sample $\mathrm{D}_{\mathrm{s}}$ (respect., sample $\mathrm{D}_{\mathrm{PnC}}$ ) at resonance. The sign of the displacements has been manually applied. The shape of the displacements distribution indicates that at resonance the modal vibration for both samples is the sixth order flexural mode, perhaps mixed with an additional slight lateral U-shaped vibration (which is more pronounced for $\mathrm{D}_{\mathrm{PnC}}$ ). The use of the 3 periods PnC strip therefore increases the quality factor Q by a factor of 27 . Note that another interesting mode, mainly flexural, is also in the bang gap. Its quality factor without the $\mathrm{PnC}$ strip is 130 and more than 1800 with the PnC. Therefore, the increase is around 15. For all the modes in the band gap where the losses from the anchor are predominant, the use of a phononic crystal increases the quality factor. Indeed, the PnC reduces the energy leak by reducing the elastic waves leaving the structure and going into the substrate. As $Q$ is the ratio of stored energy to energy dissipation, decreasing the energy leak increases $\mathrm{Q}$. The increase of the quality factor depends on the anchor losses compared to the others losses (in our case, the air damping is the cause of the second most important losses for $D_{s}$ and the first for $D_{P n C}$ ). Working under vacuum would lead to an even better increase. As long as we can work with the usual approximation of continuous media with linear elastic equations, the band gap computation can be normalized with the PnC lattice constant $a$. Decreasing the overall dimensions of our devices to work at higher frequencies will lead to similar results although the proportions of the different losses may change. It should also be noted that imperfections in the fabrication due to a reduction of the size may reduce the band gap.

In order to confirm that the increase in $\mathrm{Q}$ is indeed due to a decrease of anchor losses induced by the PnC strip, additional FEM simulations were performed. The supporting base was modeled as a half disk with a $3 \mathrm{~mm}$ diameter and a $750 \mu \mathrm{m}$ thickness. It was surrounded by a $1.5 \mathrm{~mm}$ wide perfectly matched layer (PML). We added air damping loss by including a cylinder of air with a $900 \mu \mathrm{m}$ diameter around the cantilever and a half sphere at its end. 
Cylindrical and spherical wave radiation were used as boundary conditions, respectively. In order to increase the mesh resolution, we simulated only half of the samples, using symmetries. The cases with or without the PnC strip were compared with an eigenfrequency search around $2 \mathrm{MHz}$. The quality factors of the modes are then given by half the ratio of the real part of the eigenfrequency $f_{i}$ to its imaginary part [27]:

$$
Q_{i}=\frac{\operatorname{Re}\left(f_{i}\right)}{2 \times \operatorname{Im}\left(f_{i}\right)} \text {. }
$$

The obtained quality factors for the sixth order flexural mode with and without the PnC strip are respectively 40 and 4140 . Computing the frequency response with a harmonic edge load excitation placed at the end of the cantilever leads to results of the same order of magnitude. Although the numerical $Q$ values are within a factor of 2 of the experimental values, the orders of magnitude are consistent. Removing the air loss in the numerical simulation leads to a value of the quality factor that is very sensitive to the mesh for sample $\mathrm{D}_{\mathrm{PnC}}$ and that is larger than $10^{5}$ (the imaginary part of the eigenfrequency is near zero for the best meshes). These simulations thus confirm that the experimental increase in the quality factor is mostly due to the reduction of anchor loss and that performing experiments under a vacuum would probably increase the quality factor. It should be noted that further numerical simulations showed that losses from thermoelastic damping (TED) are negligible in the case considered.

To verify the effect of the number of periods of the $\mathrm{PnC}$, we also performed numerical simulation for 1, 2 and 4 unit cells. Figure 4 summarizes the results. Adding a fourth period to the PnC provides one with only a small increase in the quality factor. Indeed, it has been previously shown that the effectiveness of PnCs in enhancing Q plateaus after three PnC cells $[17,22]$. Using a three-period PnC hence seems to be a good compromise with respect to efficiency and size.

As a conclusion, we have demonstrated the effectiveness of using a PnC strip to increase the quality factor of a flexural mode of a cantilever by a factor of 27 . Numerical simulations support the statement that the increase in $\mathrm{Q}$ is due to reduction of anchor losses. The fabrication of the PnC strip uses few steps of standard MEMS processes and is compatible with low-cost batch production. Confining the energy in scanning microscope probes may increase their efficiency. Indeed the signal to noise ratio is directly linked to the quality factor. Adding PnC strips to local probes should in this respect provide anchor loss reduction. Furthermore, in this work, the piezoelectric excitation at the base is not optimum since the $\mathrm{PnC}$ reduced the energy transmitted to the tip. A well-adapted source excitation, for instance applied directly on the tip with photoelastic excitation, would then increase the energy stored in the cantilever, leading to an improvement of the sensitivity. The design of this $\mathrm{PnC}$ is robust against imperfections in the fabrication, not fragile and uses standard CMOS compatible MEMS techniques. It can be used to shield or confine elastic energy (with frequencies in the band gap) and therefore can also be another possibility to reduce anchor loss in MEMS resonators and increase their quality factors.

This work was supported by the EIPHI Graduate School (grant number ANR-17-EURE-0002) and the French "Investissements d'Avenir" program, project ISITE-BFC (contract ANR-15IDEX-0003). The authors acknowledge support of the French RENATECH network and its FEMTO-ST technological facility MIMENTO. They would also like to thank Sarah Benchabane-Gaiffe for the use of the interferometer and Vincent Laude for fruitful discussions.

\section{DATA AVAILABILITY}

The data that support the findings of this study are available from the corresponding author upon reasonable request. 

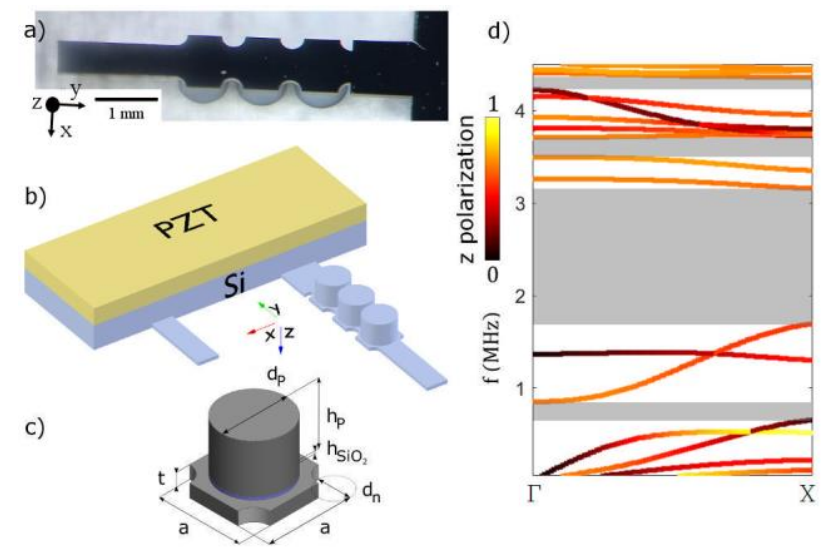

Figure 1: (a) Photograph of the cantilever with the 3 periods PnC strip ( $\left.\mathrm{D}_{\mathrm{PnC}}\right)$. (b) Schematic of the samples. (c) Unit cell of the PnC strip. (d) Phononic band structure of the PnC strip. The color bar indicates the out-of-plane $\mathrm{z}$ polarization. The gray areas indicate the band gaps.

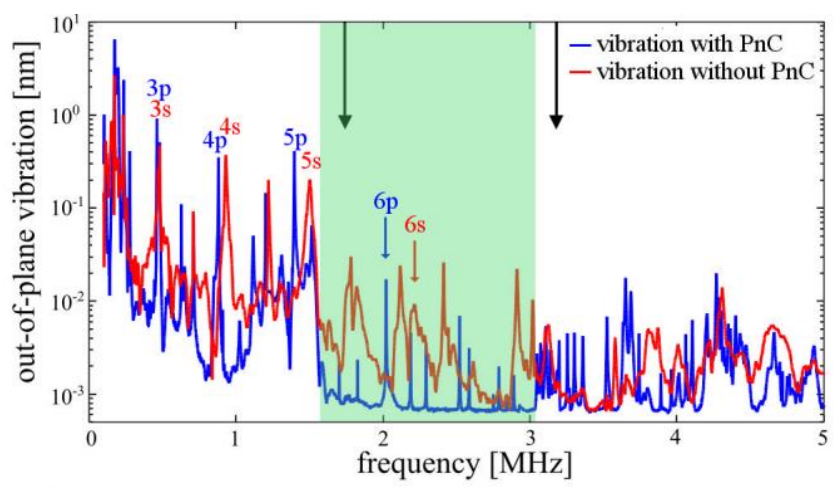

Figure 2: Experimental spectrum of the out-of-plane vibration of $\mathrm{D}_{\mathrm{s}}$ and $\mathrm{D}_{\mathrm{PnC}}$ averaged on the surface of the cantilever obtained from the MSA 500 laser Doppler vibrometer. Blue and red arrows points to the resonances of figure 3. Flexural modes (third to sixth) are indicated ( $p$ for with PnC, $s$ for without PnC). The black arrows at the top show the start and the end of the theoretical band gap whereas the green area shows the experimental one.

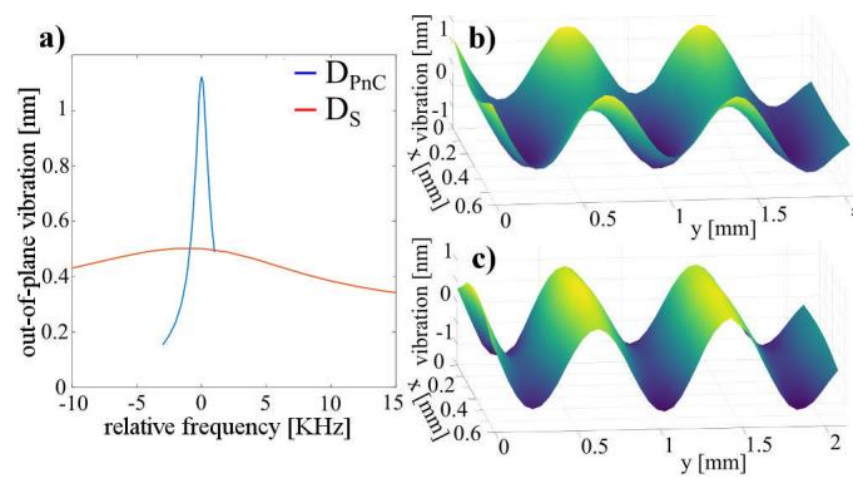

Figure 3: (a) Experimental spectrum of the out-of-plane vibration of Ds and $\mathrm{D}_{\mathrm{PnC}}$ averaged over the surface of the cantilever. Maps of the experimental out-of-plane vibration for samples $D_{s}$ (b) and $\mathrm{D}_{\mathrm{PnC}}$ (c) are shown, where the sign of the vibration has been manually applied. 


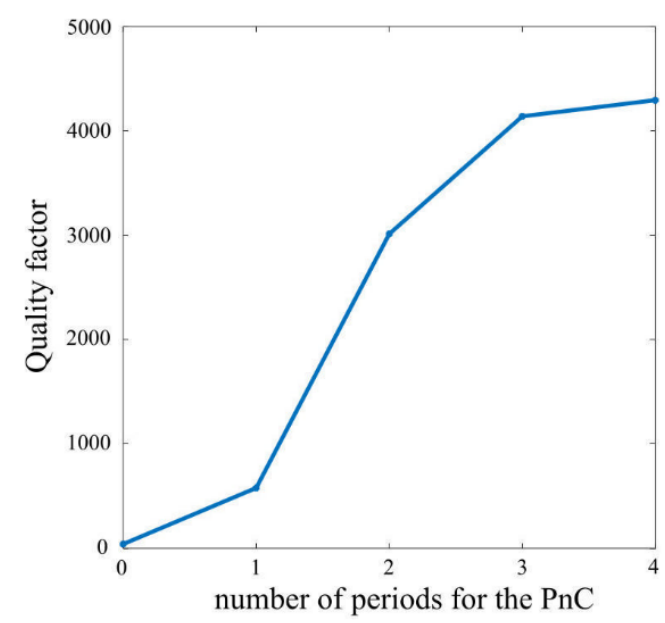

Figure 4: Simulated quality factor according to the number of periods for the phononic crystal strip.

\section{REFERENCES}

[1] M. S. Kushwaha, P. Halevi, L. Dobrzynski, and B. Djafari-Rouhani, Phys. Rev. Lett. 71, 2022, (1993). https://doi.org/10.1103/PhysRevLett.71.2022

[2] M. Sigalas and E. Economou, Solid State Communications 86, 141 (1993). https://doi.org/10.1016/0038-1098(93)90888-T

[3] D. Lee, J. H. Oh, I. S. Kang, and J. Rho, Phys. Rev. E 100, 063002, (2019). https://doi.org/10.1103/PhysRevE.100.063002

[4] M. Nomura, Microsyst Technol (2016) 22:473-480, doi: 10.1007/s00542-015-2569-5

[5] Y. Pennec, B. Djafari-Rouhani, J. O. Vasseur, A. Khelif, and P. A. Deymier, Phys. Rev. E 69, 046608 (2004). https://doi.org/10.1103/PhysRevE.69.046608

[6] M. Hussein, K. Hamza, G. Hulbert, R. Scott, and K. Saitou, Struct Multidisc Optim 31, 6075 (2006). https://doi.org/10.1007/s00158-005-0555-8

[7] D. Yu, J. Wen, H. Zhao, Y. Liu, and X. Wen, Journal of Sound and Vibration 318, 193 (2008), https://doi.org/10.1016/j.jsv.2008.04.009

[8] J. Christensen, A. Fernandez-Dominguez, F. De Leon-Perez, L. Martin-Moreno, and F. Garcia-Vidal, Nature Physics 3, 851 (2007). https://doi.org/10.1038/nphys774

[9] M. S. Kushwaha, Appl. Phys. Lett. 70, 3218 (1997). https://doi.org/10.1063/1.119130

[10] C. Goffaux, F. Maseri, J. O. Vasseur, B. Djafari-Rouhani, and P. Lambin, Applied Physics Letters 83, 281 (2003). https://doi.org/10.1063/1.1592016

[11] A. Khelif, A. Choujaa, S. Benchabane, B. Djafari-Rouhani, and V. Laude, Applied Physics Letters 84, 4400 (2004). https://doi.org/10.1063/1.1757642

[12] J. O. Vasseur, A.-C. Hladky-Hennion, B. Djafari-Rouhani, F. Duval, B. Dubus, Y. Pennec and P. A. Deymier, Journal of Applied Physics 101, 114904 (2007). https://doi.org/10.1063/1.2740352

[13] M.-J. Chiou, Y.-C. Lin, T. Ono, M. Esashi, S.-L. Yeh, and T.-T. Wu, Ultrasonics 54, 1984 (2014). https://doi.org/10.1016/j.ultras.2014.05.007

[14] F.-C. Hsu, J.-C. Hsu, T.-C. Huang, C.-H. Wang, and P. Chang, J. Phys. D: Appl. Phys. 44, 375101 (2011). https://doi.org/10.1088/0022-3727/44/37/375101

[15] L. Sorenson, J. Fu, and F. Ayazi, in Solid-State Sensors, Actuators and Microsystems Conference (TRANSDUCERS), 2011, 16th International (2011), pp. 918-921. https://doi.org/10.1109/TRANSDUCERS.2011.5969685

[16] D. Feng, D. Xu, G. Wu, B. Xiong, and Y. Wang, J. Appl. Phys. 115, 024503 (2014). https://doi.org/10.1063/1.4861409

[17] H. Zhu and J. E.-Y. Lee, Procedia Engineering 120, 516 (2015), eurosensors 2015. https://doi.org/10.1016/j.proeng.2015.08.689 
[18] H. Zhu and J. E.-Y. Lee, 2015 28th IEEE International Conference on Micro Electro Mechanical Systems (MEMS), https://doi.org/10.1109/MEMSYS.2015.7051079

[19] L. Binci, C. Tu, H. Zhu and J. E.-Y. Lee, Appl. Phys. Lett 109, 203501 (2016). https://doi.org/10.1063/1.4967794

[20] V. J. Gokhale and J. J. Gorman, Appl. Phys. Lett. 111, 013501 (2017). https://doi.org/10.1063/1.4990960

[21] F.-H. Bao, L.-L.i Bao, X.-Y. Li, M. A. Khan, H.-Y. Wu, F. Qin, T. Zhang, Y.Zhang, J.-F. Bao, and X.-S. Zhang, Applied Physics Express 11, 067201 (2018); doi: 10.7567/APEX.11.067201

[22] M. W. U. Siddiqi and J. E.-Y. Lee, Micromachines 2018, 9(8), 413; doi: 10.3390/mi9080413

[23] E. Coffy, T. Lavergne, M. Addouche, S. Euphrasie, P. Vairac, and A. Khelif, Journal of Applied Physics 118, 214902 (2015); doi: 10.1063/1.4936836

[24] E. Coffy, S. Euphrasie, M. Addouche, P. Vairac, A. Khelif, Ultrasonics 78, 51-56 (2017); doi: 10.1016/j.ultras.2017.03.003

[25] P. Vairac, and C. Crétin, Opt. Comm. 132, 19 (1996); doi: 10.1016/0030-4018(96)003264

[26] S. Benchabane, O. Gaiffe, R. Salut, G. Ulliac, V. Laude, and K. Kokkonen, Appl. Phys. Lett. 106, 081903 (2015); doi: 10.1063/1.4913532

[27] T. D. Ha, J.F. Bao, Microsyst. Technol. 22, 791 (2016), doi: 10.1007/s00542-015-2678-1 